\title{
Moving from the Oslerian paradigm to the post- genomic era: are asthma and COPD outdated terms?
}

\author{
Lowie E G W Vanfleteren, 1,2 Janwillem W H Kocks, 3,4 lan S Stone, 5,6 \\ Robab Breyer-Kohansal, ${ }^{7}$ Timm Greulich, ${ }^{8}$ Donato Lacedonia, ${ }^{9}$ Roland Buhl, ${ }_{1}^{10}$ \\ Leonardo M Fabbri, ${ }^{11}$ Ian D Pavord, ${ }^{12}$ Neil Barnes, ${ }^{6}$ Emiel F M Wouters, ${ }^{1,2}$ \\ Alvar Agusti ${ }^{13}$
}

For numbered affiliations see end of article.

\section{Correspondence to Dr Lowie Vanfleteren, Program Development, CIRO+, A Centre of Expertise for Chronic Organ Failure, Hornerheide 1, Horn 6085 NM, The Netherlands; lowievanfleteren@ciro-horn.nl}

Received 20 March 2013 Revised 3 May 2013 Accepted 30 May 2013 Published Online First 22 June 2013
To cite: Vanfleteren LEGW, Kocks JWH, Stone IS, et al. Thorax 2014;69:72-79.

\section{ABSTRACT}

In the majority of cases, asthma and chronic obstructive pulmonary disease (COPD) are two clearly distinct disease entities. However, in some patients there may be significant overlap between the two conditions. This constitutes an important area of concern because these patients are generally excluded from randomised controlled trials (mostly because of smoking history in the case of asthma or because of significant bronchodilator reversibility in the case of (OPD). As a result, their pathobiology, prognosis and response to therapy are largely unknown. This may lead to suboptimal management and can limit the development of more personalised therapeutic options. Emerging genetic and molecular information coupled with new bioinformatics capabilities provide novel information that can pave the way towards a new taxonomy of airway diseases. In this paper we question the current value of the terms 'asthma' and 'COPD' as still useful diagnostic labels; discuss the scientific and clinical progress made over the past few years towards unravelling the complexity of airway diseases, from the definition of clinical phenotypes and endotypes to a better understanding of cellular and molecular networks as key pathogenic elements of human diseases (so-called systems medicine); and summarise a number of ongoing studies with the potential to move the field towards a new taxonomy of airways diseases and, hopefully, a more personalised approach to medicine, in which the focus will shift from the current goal of treating diseases as best as possible to the so-called P4 medicine, a new type of medicine that is predictive, preventive, personalised and participatory.

\section{RATIONALE: WHY ASKING?}

Sir William Osler, arguably the founder of modern medicine, established in the nineteenth century the current classification of diseases by linking the principal organ system in which symptoms and signs manifest with anatomic and histopathology findings. ${ }^{1}$ To date, this Oslerian paradigm has been extremely helpful as it establishes syndromic patterns that limit the number of potential pathophenotypes clinicians may need to consider. Over the past few decades, however, a number of significant advances in molecular medicine, including the successful sequencing of the entire human genome, ${ }^{2}$ led to an explosion of new molecular knowledge that raised awareness progressively about the imprecision of this traditional approach. Classifying diseases according to this Oslerian paradigm vastly overgeneralises patho-phenotypes, does not usually take into consideration susceptibility states or preclinical disease manifestations, and cannot be used to individualise disease diagnosis or therapy. As a result, it has been proposed that, in the post-genomic era and in order to progress towards personalised medicine, diseases will have to be reclassified bottom up (from molecules to symptoms) in contrast to the classical top-down classification initiated by Osler. ${ }^{3}$

Airways diseases do not escape this debate. In particular, asthma and chronic obstructive pulmonary disease (COPD), the two most common airway diseases, are sometimes difficult to distinguish using the traditional Oslerian approach, whereas emerging genetic and molecular information can provide novel information for a better taxonomy of airway diseases. In this paper we question the current value of the terms 'asthma' and 'COPD' as still useful diagnostic labels in these patients; discuss the scientific and clinical progress made over the past few years towards unravelling the complexity of airway diseases, from the definition of clinical phenotypes and endotypes to a better understanding of cellular and molecular networks as key pathogenic elements of human diseases (so-called systems medicine); and summarise the potential to move the field towards a new taxonomy of airways diseases and, hopefully, a more personalised approach to medicine, in which the focus will shift from the current goal of treating diseases as best as possible to the so-called P4 medicine, a new type of medicine that is predictive, preventive, personalised and participatory.

\section{THE TRADITIONAL APPROACH: WHERE ARE WE NOW?}

The currently accepted definitions of asthma and COPD are shown in box 1. In the large majority of cases, asthma and COPD are two clearly distinct disease entities. In general, asthma develops in childhood, mostly in atopic subjects, is most prevalent in adolescents and young adults, develops in nonsmokers, and manifests itself with recurrent episodes of acute airway obstruction with normal lung function in between. By contrast, COPD is diagnosed in subjects older than 40 years, is most prevalent in older people, develops in smokers and/or subjects exposed to indoor or outdoor pollution, manifests itself with chronic persistent symptoms that occasionally exacerbate, and is associated with non-reversible 


\section{Box 1 Current definition of asthma and COPD}

- Asthma is a chronic inflammatory disorder of the airways in which many cells and cellular elements play a role. The chronic inflammation is associated with airway hyper-responsiveness that leads to recurrent episodes of wheezing, breathlessness, chest tightness, and coughing, particularly at night or in the early morning. These episodes are usually associated with widespread, but variable, airflow obstruction within the lung that is often reversible either spontaneously or with treatment (GINA $2011^{5}$ )

- COPD, a common preventable and treatable disease, is characterised by persistent airflow limitation that is usually progressive and associated with an enhanced chronic inflammatory response in the airways and the lung to noxious particles or gases Exacerbations and comorbidities contribute to the overall severity in individual patients (GOLD 2011 ${ }^{4}$ )

COPD, chronic obstructive pulmonary disease; GOLD, Global Initiative for Chronic Lung Diseases.

airflow limitation due to both airway (bronchiolitis) and lung parenchymal (emphysema) abnormalities.

Despite this apparently clear distinction between asthma and COPD, there is significant overlap between the two conditions, as recently acknowledged by the Global Initiative for Chronic Lung Diseases (GOLD) ${ }^{4}$ and the Global Strategy for Asthma Management and Prevention (GINA). ${ }^{5}$ Terms such as 'airway disease', 'inflammatory disease' and 'airflow limitation/obstruction' are used by both definitions. The most common symptoms of asthma and COPD (dyspnoea, cough, wheezing) are similar. Lung function abnormalities can also be remarkably similar since reversible airflow limitation, the key defining feature of asthma, may be present in a substantial proportion of subjects with COPD. ${ }^{6}$ Alternatively, an accelerated decline in lung function, a supposedly key defining feature of COPD, has been seen in patients with asthma who have exacerbations and who do not take inhaled steroids $^{7}$ and in patients with asthma who smoke. ${ }^{8}$ Both diseases also share numerous risk factors, including the genetic background of the individual and family, environmental exposures (such as respiratory infections and diet during childhood, active and passive smoking and other exposures) and deprived socioeconomic status, all of them with the potential to modulate maximally attained lung function in adolescence and to influence respiratory disease later in life. ${ }^{5}{ }^{9}$ Interestingly, the pathology of the airway and lung, albeit clearly different in classical asthma and COPD, shows significant similarities in some cases. For instance, a role for mast cells, a major player in asthma, has been described in centri-lobular emphysema in which mast cells were predominant in the smooth muscle of small airways and the alveolar walls of these patients and were related to the degree of airway reactivity. ${ }^{10}$

In clinical practice there is a substantial group of adults with asthma or COPD who may share similar clinical characteristics. This constitutes an important area of concern because its pathobiology is largely unknown, is often unrecognised and is frequently poorly treated. For instance, these patients are often excluded from randomised controlled trials because of smoking history in the case of asthma or because of significant bronchodilator reversibility in the case of COPD, thus limiting the generalisability of clinical trial results.
Attempts to define asthma and COPD based on a change in lung function as the main (and often only) surrogate has resulted in current definitions failing to recognise their complexity, leading to suboptimal disease classification and management, with subsequent difficulties in drug development, since airway disorders with different pathobiology may require different therapeutic strategies. For example, eosinophilic airway inflammation is causally associated with exacerbations of asthma and COPD, but it is not associated with airway hyperresponsiveness. ${ }^{11}$ However, for 10 years monoclonal antibodies inhibiting interleukin 5 (ie, mepolizumab), which are specific and effective inhibitors of eosinophilic airway inflammation, were tested exclusively (and unsuccessfully) in asthma against outcomes related to airway hyper-responsiveness in patients identified by the presence of variable airflow obstruction and/or airway hyper-responsiveness (and not by the presence of eosinophilic airway inflammation). ${ }^{11}$ When mepolizumab was evaluated in patients with the latter against an outcome that was associated with eosinophilia (exacerbations), efficacy was clearly established on this specific outcome. ${ }^{12}$ The benefits of treatment were least in those with a large response to bronchodilator therapy, a population that could be regarded as having the most 'asthma' and there was clear evidence of efficacy in patients with features of 'COPD', such as adult onset disease and fixed airflow obstruction. Interestingly, eosinophilic airway inflammation often contributes to exacerbations of COPD ${ }^{13}$ and it is generally poorly responsive to inhaled corticosteroids. ${ }^{14}$ Patients with eosinophilic COPD therefore have an important unmet need for a specific inhibitor of eosinophilic airway inflammation free of the side effects of oral corticosteroids. Regrettably regulatory agencies still insist on the use of traditional markers of variable airflow obstruction to identify patients and measure the efficacy of anti-eosinophil drugs.

It is clear that the 'definitions' of asthma and COPD provided in the boxes are merely descriptions; it is not clear how many characteristics are needed to make a diagnosis. These essentialist definitions convey the message that asthma and COPD are separate disease entities, but they are long winded and fail to identify a primary defining characteristic which separates them from each other and other diseases. The alternative approach that overcomes these disadvantages is to use nominalist definitions that identify single defining characteristics to make a diagnosis. Objective measurements will be required at different levels considering all components of airway disease for an accurate diagnosis. The current diagnostic labels asthma and COPD would fall apart into separate nominalist definitions. ${ }^{15}$

\section{HOW CAN WE PROGRESS FROM HERE?}

Over the past decade, extensive research into asthma and COPD has led to significant improvements in our understanding and management of both diseases. Yet, this new knowledge has generally been interpreted 'top-down', according to the Oslerian disease classification. Biological complexity can only be addressed by unravelling the parts that form the system, and their inter-relationships, a strategy embraced by the new disciplines of systems biology and network medicine. ${ }^{16}$ This 'bottom-up' strategy has the potential to facilitate the development of better targeted, more appropriate, more effective and safer therapies for the treatment of different subgroups of patients with airway disease, unlocking the full potential of personalised medicine. ${ }^{3}$ Below we discuss a number of different (but complementary) research strategies based on this new vision. 


\section{Clinical phenotypes}

A recent consensus definition of a 'clinical phenotype' has been proposed (box 2). ${ }^{17} 18$ This definition emphasises two key aspects: the attribute(s) that define the clinical phenotype cannot occur in all patients with the disease of interest, since they have to identify a subgroup of patients that differ in their prognosis and/or therapeutic needs; and these attributes (hence, the clinical phenotype itself) have to relate to clinically meaningful outcomes, such as symptoms, exacerbations, response to therapy, rate of disease progression and/or death. This implies that any 'potential' clinical phenotype must be validated longitudinally against these outcomes and that, before this, these 'phenotypic traits' should be considered as mere descriptors of the complexity of the disease. ${ }^{19}$ In this context, it is of interest to review the asthma and COPD 'clinical phenotypes' that have been validated to date.

In COPD, $\alpha 1$-antitrypsin deficiency is probably the best example of a well validated clinical phenotype in COPD, since the genetic defect is well described, it is easily diagnosed with a serum biomarker, and it may require specific supplementation therapy. ${ }^{20}$ Other validated COPD clinical phenotypes include patients with the presence of upper lobe emphysema combined with low exercise capacity after rehabilitation, which is an indication for lung volume reduction surgery ${ }^{21}$; the 'frequent exacerbator' phenotype, ${ }^{22}$ a condition which is now included in the new GOLD 2011 assessment proposal ${ }^{4}$ because of its potential therapeutic implications ${ }^{23-25}$; and finally, the presence of persistent systemic inflammation, which is associated with high mortality in the short term. ${ }^{26}$

In asthma, 'classic' early onset atopic asthma could be seen as a distinct clinically important phenotype because it is generally associated with an excellent response to inhaled corticosteroid therapy. Most of these patients are likely to have been included in previous asthma trials and are likely to be found in the 'concordant disease' cluster proposed by Haldar et $a l^{27}$ or the 'classical asthma phenotype' described by Wardlaw et $a l^{28}$ or the 'milder asthma' cluster in the study by Moore et al ${ }^{29}$ Other well described clinical phenotypes in asthma include aspirin-intolerant asthma; obesity-related asthma, which occurs primarily in older women, and is highly symptomatic but less often associated with bronchial hyper-responsiveness ${ }^{19}{ }^{30}$; late onset eosinophilic asthma, which develops later in life, is less allergic, more corticosteroid refractory but responsive to antileukotrienes and mepolizumab ${ }^{12}{ }^{19}$; neutrophilic asthma, associated with lower lung function and (most probably)

\section{Box 2 Phenotypes and clinical phenotypes}

- A phenotype is 'any observed quality of an organism, such as its morphology, development or behaviour, as opposed to its genotype-the inherited instructions it carries, which may or may not be expressed'. The phenotype is composed of traits or characteristics, some of which are controlled entirely by the individual's genes, whereas others are controlled by genes but are significantly affected by environmental factors. ${ }^{\prime 17}$

- A clinical phenotype is 'a single or combination of disease attributes that describe differences between individuals as they relate to clinically meaningful outcomes (symptoms, exacerbations, response to therapy, rate of disease progression, or death. ${ }^{\prime 18}$ responsive to macrolides, ${ }^{19}{ }^{31}$ although this phenotype is probably less distinct since the use of inhaled corticosteroids can increase neutrophilic inflammation in some patients. ${ }^{32}$ The latter two phenotypes are generally recognised at older age ('late onset') and may be difficult to differentiate from COPD. Indeed, as discussed above, eosinophilia is frequently an important contributor to COPD exacerbations ${ }^{13}$ and macrolides can reduce exacerbations in asthma and COPD. ${ }^{6} 31$

Recognising the overlap between asthma and COPD, Pavord and Wardlaw proposed to return to the term 'airway diseases' and to use a classification system (A, B, C, D and E) based on Airway hyper-responsiveness, responsible for short-term variable bronchodilator responsive airflow obstruction, Bronchitis, which may be eosinophilic and corticosteroid responsive or neutrophilic and corticosteroid resistant, Cough reflex hypersensitivity, Damage to the airway and lung resulting in fixed airflow obstruction, impaired gas transfer, emphysema, bronchiectasis, and extrapulmonary factors such as dysfunctional breathing, adherence to medication, deconditioning, weight loss or gain, muscle weakness, premature vascular disease. ${ }^{33}$

\section{Endotypes (or intermediate pathophenotypes)}

The biological armamentarium of human biology to respond to injury is limited and includes inflammation, thrombosis/haemorrhage, fibrosis, immune response, cell proliferation and cell death (apoptosis/necrosis). ${ }^{3}$ These so-called intermediate pathophenotypes or endotypes (a contraction of endophenotypes), alone or in combination, determine variation in the clinical presentation of diseases in individuals with similar underlying genetic and/or environmental exposures. ${ }^{3}$ A number of different endotypes have been proposed in asthma ${ }^{34}$ and COPD, ${ }^{35}$ including alterations in the innate and acquired immune response, tissue repair, remodelling, accelerated aging and senescence, oxidative stress, enhanced apoptosis and defective catabasis (figure 1). A better understanding of these endotypes might facilitate the development of new molecular treatments such as novel biological therapy alternatives like anti-IgE (omalizumab), ${ }^{36}$ anti-interleukin-13 (IL13) (lebrikizumab) ${ }^{37}$ and anti-IL5 (mepolizumab). ${ }^{12}$ The use of these therapeutic alternatives, however, requires the validation of biomarkers, such as $\operatorname{IgE}$ or periostin serum levels, ${ }^{37}$ that help to identify those patients who can better respond to them. Likewise, techniques that recognise the molecular signature of exhaled breath with the use of an 'electronic nose' may also potentially contribute to differentiating between different airway disease phenotypes related to specific endotypes. ${ }^{38}$

\section{An unbiased approach}

The advantage of 'the phenotype approach' discussed above is that 'new' phenotypes are based on current knowledge, making implementation into practice more feasible. However, precisely because of this, it is unlikely that this approach can discover entirely 'new' phenotypes. An alternative strategy is to work without pre-assumptions using an unbiased, 'hypothesisgenerating' or 'discovery-driven' approach. In this way, 'patterns' of clinical and/or biological features can be detected with the use of statistical methods, such as cluster analysis and principal component analysis, that automatically (ie, in an unbiased manner) group 'similar' patients into 'independent' clusters. Yet, several methodological limitations are of note. First, the population in which the unbiased approach is applied might already be biased by the inclusion of subjects who have an Oslerian 'disease classification label' (asthma or COPD). In fact, to date, only one study has included a random population 


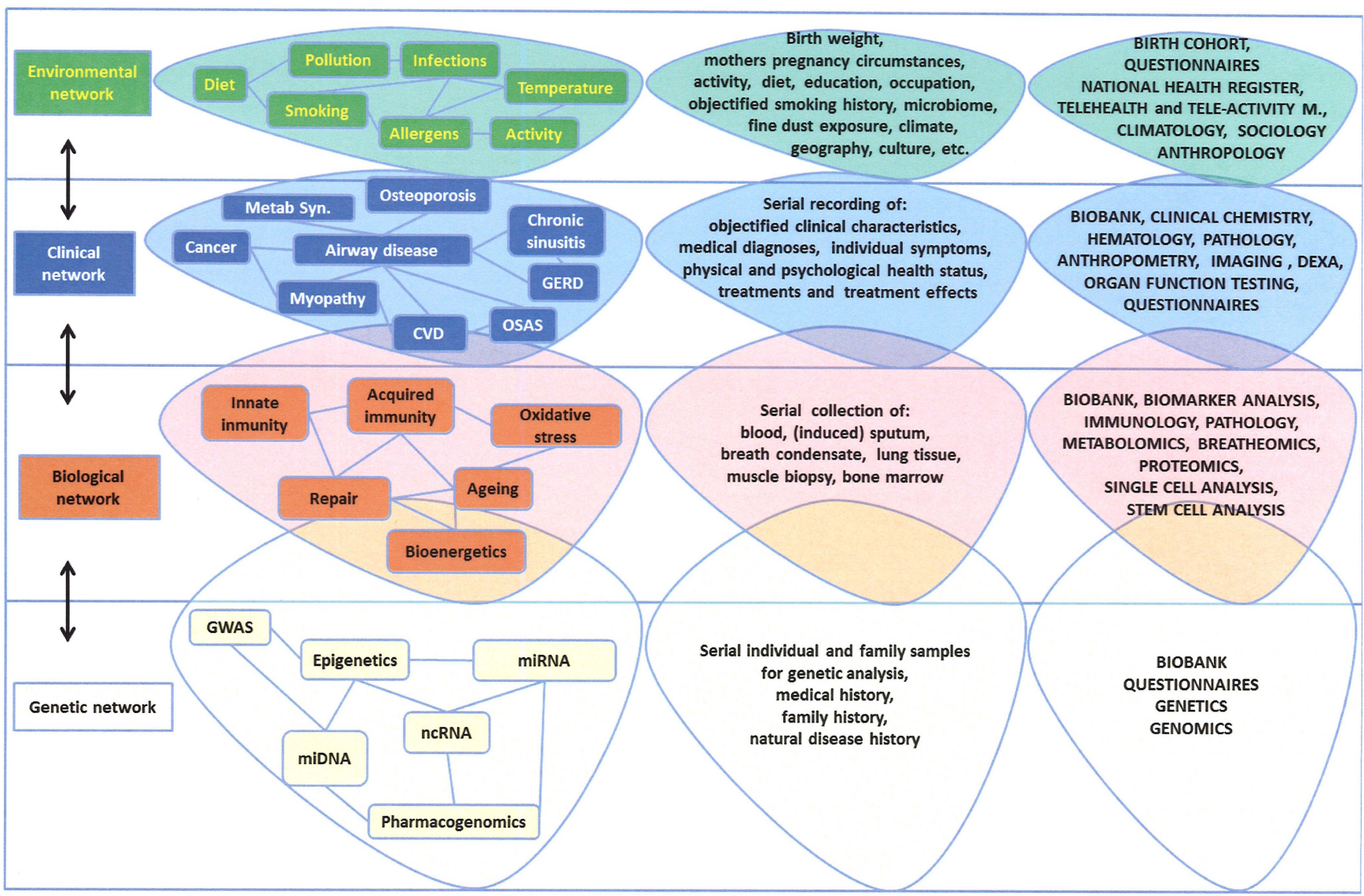

Figure 1 Network medicine in airway disease (modified with permission from Agusti and Vestbo ${ }^{35}$ ). Diagram illustrating the different levels of complexity of airway disease. At each level, only some of the potential components are shown to illustrate the concept (the diagram is not intended to be comprehensive). Likewise, links between the different elements of the network are drawn for illustrative purposes only and do not necessarily reflect evidence-based relationships. The middle and right-hand column respectively show the data required and the logistics and scientific knowledge needed to perform a study on a new taxonomy of airway disease. For further explanations, see text. CVD, cardiovascular disease; GERD, gastroesophageal reflux disease; GWAS, genome-wide association studies; M, monitoring; miDNA, mitochondrial DNA; miRNA, microRNA; ncRNA, noncoding RNA; OSAS, obstructive sleep apnoea syndrome.

sample of patients with airway symptoms or spirometric abnormalities without a prespecified label of asthma or COPD. ${ }^{39}$ Interestingly, among the five identified clusters, 'Oslerian'-defined diseases such as classic allergic asthma were identified. Second, the identified clusters depend directly on the type and amount of information included in the analysis. Third, the number of expected clusters can be set manually, thus influencing the results and removing the label 'unbiased' partially. Fourth, in practice, it is difficult to prospectively determine which cluster an individual belongs to. Finally, unless the identified clusters are associated with relevant outcomes, such as mortality or treatment requirements, their clinical relevance or validity is unclear.

To date, this unbiased approach has been used in several asthma and COPD studies. Only one of them, ${ }^{40}$ however, validated the relationship of the identified clusters with clinically relevant longitudinal outcomes. Garcia-Aymerich et al studied patients with COPD who required hospitalisation because of an exacerbation of the disease for the first time and identified three different clusters with marked clinical differences (severe respiratory COPD, moderate respiratory COPD and a group with milder respiratory characteristics but more obesity, cardiovascular and metabolic disease) that, importantly, were associated with different mortality and hospitalisation requirements during follow-up. ${ }^{40}$ More recently, Vanfleteren $e t ~ a l^{41}$ identified five distinct COPD clusters based on the type of comorbidity present ('less comorbidity'; 'cardiovascular'; 'cachectic'; 'metabolic'; and 'psychological') that were associated with markedly different health status despite similar COPD severity.

\section{The network/systems approach}

Traditionally, only lung function measurements, such as the degree of airflow limitation (forced expiratory volume in $1 \mathrm{~s}$ $\left(\mathrm{FEV}_{1}\right)$ ) or peak flow variability, have been used to describe the severity of COPD and asthma. More recently, with increasing realisation of the complexity of both diseases, multicomponent indexes have been developed. In the case of COPD, these include the BODE (body mass index, $\mathrm{FEV}_{1}$, dyspnoea, and exercise capacity) index, ${ }^{42}$ the $\mathrm{ADO}$ (age, dyspnoea, $\mathrm{FEV}_{1}$ ) index, ${ }^{43}$ and the DOSE (dyspnoea, $\mathrm{FEV}_{1}$, smoking status, and frequency of exacerbation) index. ${ }^{44}$ In the case of asthma, severity is generally classified on the basis of the intensity of treatment required to achieve good asthma control. ${ }^{5}$ More recently, the Composite Asthma Severity Index, based on daytime and nocturnal symptoms, albuterol use, controller treatment, lung function measures and exacerbations, was introduced to measure 
Table 1 Summary of ongoing research initiatives, in Europe and the USA, aiming at a better understanding of the complexity of asthma and COPD

\begin{tabular}{|c|c|}
\hline Study name & Goal \\
\hline $\begin{array}{l}\text { U-BIOPRED } \\
\text { Unbiased Biomarkers for the } \\
\text { Prediction of Respiratory Disease } \\
\text { Outcomes } \\
\text { http://www.ubiopred.eu }\end{array}$ & $\begin{array}{l}\text { To better understand the different types } \\
\text { of severe asthma with the hope that this } \\
\text { understanding will consider individual } \\
\text { characteristics and make it easier to } \\
\text { develop new medicines }\end{array}$ \\
\hline $\begin{array}{l}\text { EvA } \\
\text { Emphysema versus Airway study } \\
\text { http://www.eva-copd.eu }\end{array}$ & $\begin{array}{l}\text { To explore the concept that emphysema } \\
\text { and airway disease are governed by } \\
\text { different pathophysiological processes, } \\
\text { are driven by different genes and have } \\
\text { differential gene expression in the lung }\end{array}$ \\
\hline $\begin{array}{l}\text { MeDALL } \\
\text { Mechanisms of the Development } \\
\text { of Allergy } \\
\text { http://www.medall-fp7.eu }\end{array}$ & $\begin{array}{l}\text { To generate novel knowledge on the } \\
\text { mechanisms of initiation of allergy from } \\
\text { early childhood to young adulthood to } \\
\text { propose early diagnosis, prevention and } \\
\text { targets for therapy }\end{array}$ \\
\hline
\end{tabular}

Type of patients included

500 adult patients with severe asthma and several control groups, including up to 75 patients with non-severe asthma, 100 patients with COPD and 50 non-smoking healthy people

500 patients with COPD (ex-smokers) and 300 healthy volunteers (age $>45$, never smokers or ex-smokers)

Large network of existing birth cohorts

Methods and tools used in systems biology will be applied to facilitate an effective knowledge management strategy and to integrate the findings of the different mechanistic studies built up on the samples provided by the birth cohorts. The information from the mechanistic studies will be used to validate the classical and novel phenotypes and to assess interactions with relevant environmental factors

Synergy-COPD project http://www.synergy-copd.eu

\section{AirPROM}

Airway Disease Predicting Outcomes through Patient Specific Computational Modelling http://www.airprom.eu

\section{ESCAPE}

European Study of Cohorts for Air Pollution Effects http://www.escapeproject.eu

\section{CHANCES}

Consortium on Health and Ageing: Network of Cohorts in Europe and the USA http://www.chancesfp7.eu

COPDgene study http://www.copdgene.org

\section{SPIROMICS}

Subpopulations and intermediate outcome measures in COPD study

http://www.cscc.unc.edu/spir/

MESA

Multi-Ethnic Study of

Atherosclerosis

http://www.mesa-nhlbi.org
To study the underlying mechanisms of COPD phenotypes associated with poor prognosis, by simulating different functions of the human body using computer models at different levels (sub-cellular, tissue, organ and organ system) The project seeks to produce a more complete computer model of the

To develop a patient-specific multiscale predictive computational airway model that can predict the development of asthma or COPD in an individual patie

Investigate long-term effects of air pollution on perinatal health outcomes, development of respiratory and

cardiovascular disease and cause-specific mortality

To combine and integrate ongoing cohort studies to produce an integrated approach to the study of health in older people. To generate a unique resource for additional studies on health and multi-morbidity in older people

To investigate the underlying genetic factors of COPD, to better classify COPD and understand how the disease may differ from person to person

To identify COPD subpopulations and intermediate outcome measures for future use in clinical studies. Secondary aims are to clarify the natural history of COPD, to develop bioinformatic resources and to create a database for further research

To investigate the prevalence and progression of subclinical cardiovascular disease. The extensive phenotyping of this 'cardiovascular' study have already provided insight in airways disease mechanisms of COPD

Existing databases of clinical trials and The project integrates all this information with research projects of the pharmaceutical physiological and molecular biology data that have industry regarding patients with COPD been processed for more than 30 years to obtain a unified model, which is the one that will be used to simulate the evolution of the disease in a person. First, contributing to foster convergence between basic and clinical sciences. Second, promoting the link between systems medicine and integrated care, aiming at personalised health for patients with chronic diseases

Patients with asthma, COPD and a small number of healthy controls from three large European cohorts EvA,

A broad multi-discipline approach (including expertise in physiology, radiology, image analysis, bioengineering, data harmonisation, security and ethics, computational modelling and systems biology) will enable development of an integrated multiscale airways model

Efficiently using health data already available from European cohort studies. To these studies, air pollution exposure assessment will be applied at the individual home address level of participants in each of these studies

Combining 15 European cohorts of Evaluating health issues related to ageing and ageing-related conditions. Health-related determinants comprising socio-economic factors, environmental factors, occupational exposures, lifestyle and nutritional factors. Health-related conditions comprise mainly cancer, diabetes, cardiovascular disease, bone disease and cognitive disorders but also respiratory and other diseases

10000 smokers with and without COPD across all GOLD stages

A GWAS is performed to identify genetic risk factors related to COPD. Furthermore, it is planned to characterise the disease process using high-resolution CT scans

3200 patients with COPD, 40-80 years A prospective collection and analysis of phenotypic, of age, included at six clinical centres in the USA biomarker, genetic, genomic, and clinical data, including high-resolution CT scans and bronchoscopic samples (in a subset of patients)
Population-based sample of 6814 asymptomatic men and women aged $45-84$ years
Extensive and repetitive cardiovascular functional and structural imaging, standard coronary risk factors, socio-demographic factors, lifestyle factors, and psychosocial factors. Blood samples of DNA are being extracted and lymphocytes immortalised for study of candidate genes, and possibly, genome-wide scanning 
asthma severity in children and adolescents. ${ }^{45}$ This approach is a significant step forward towards a better characterisation of disease severity, but these composite indexes lack a comprehensive biological insight. As discussed earlier in this manuscript, complexity can only be understood when unravelling the parts that comprise it and their relationships. ${ }^{16}$ This concept has been nicely illustrated by Agusti and Vestbo, ${ }^{35}$ using COPD as a use case, through the identification of four different levels (genetic, biological, clinical and environmental). Each of them requires a full understanding of the network structure but, much more challenging, we need a better knowledge of the relationships between these different levels. Most cluster analyses operate at the clinical level, while endotyping studies generally work at the biological level. Integration of these different levels (plus the genomic and environmental ones) is urgently needed. Network medicine is a new research strategy that offers a platform to explore systematically the molecular complexity of a particular disease, leading to the identification of disease modules and pathways, as well as the molecular relationships among apparently distinct (patho)phenotypes. Although still in its infancy, this strategy has the potential to allow the development of more efficient and personalised therapeutic strategies. ${ }^{16} \mathrm{~A}$ recent study illustrates the potential of this approach in COPD. Agusti et al recently described the 'systemic inflammome' of patients with COPD and showed that the 'inflammome' induced by smoking is different from that associated with COPD; contrary to what is generally thought, systemic inflammation does not occur in all patients with COPD. Actually, they provided evidence that about a third of patients have persistent inflammation. In addition, about $20 \%$ of patients with COPD have persistent inflammation, and importantly, their mortality at 3 years is six times higher than that of patients without inflammation. ${ }^{26}$ By using a simple 'network approach', the results of this study have identified a subgroup of patients with COPD who, despite similar degree of pulmonary abnormalities, have a very poor prognosis. This COPD 'phenotype' clearly deserves research to determine from which therapy it would benefit most.

\section{THE FIELD IS RAPIDLY MOVING FORWARD}

During the past few years, a number of studies in (severe) asthma (SARP, ENFUMOSA, BIOAIR) ${ }^{46}$ and COPD (ECLIPSE, PAC-COPD, CIROCO) $)^{26} 4041$ aimed at understanding the complexity of both diseases and have already produced important results, some of which have been discussed above. Yet, there are a number of ongoing research initiatives, in Europe and the USA, which will move the field forward significantly in the near future. Table 1 summarises the main aspects of these ongoing studies.

\section{TOWARDS A NEW TAXONOMY AND PERSONALISED APPROACH TO AIRWAY DISEASES}

For many patients 'asthma' and 'COPD' are outdated terms that do not fully recognise the molecular and clinical heterogeneity of chronic airway diseases. This limits the development of new and more efficient therapeutic alternatives and prevents patients from getting the best personalised medicine possible. We need a better understanding of the endotypes that underlie chronic airway diseases. This will most likely result in a new classification (taxonomy) of airway diseases. This new taxonomy should use nominalist definitions based on objectively identified single defining characteristics, more specifically based upon the characterisation of the endotypes and pattern of clinical expression (clinical phenotypes) of different airways diseases. In each of them, one or more biomarkers to be used in the clinic need to be identified and validated. Given the important influence of multiple environmental factors on the underlying endotypes, this new classification should carefully consider these environmental factors. After all, asthma and COPD are paradigmatic examples of gene-environment human diseases. To do this, a network approach that relates genes, environment, endotypes and clinical phenotypes, in an unbiased way (discovery driven, rather than hypothesis driven) will be needed. In turn, to analyse the enormous volume of information that will be acquired, new computational and analytical abilities will be needed so individual omic 'fingerprints' can be integrated to form 'handprints' that characterise clinical phenotypes. ${ }^{47}$ The functional and structural models defined at the molecular and cellular level will have to be integrated at the organ level ${ }^{47}$ and be further related to environmental factors. Figure 1 gives an overview of the interaction between the different network levels and the process by which the necessary data should be integrated. This will ultimately lead to new definitions, hence taxonomy, of airway diseases and, hopefully, to personalised medicine in which the focus of current healthcare will shift from treating diseases (reactive medicine) to the so-called P4 medicine, a new type of medicine that is predictive, preventive, personalised and participatory. ${ }^{16}$ We also have to consider the perspective of a patient who has one of these conditions and receives a long-winded phenotype/endotype/biomarker-based diagnosis. The question could arise as to whether our society is ready for this. Patients, providers, insurers and society at large need a simple diagnosis to explain their condition. However, as technology and science evolve in many parts of medicine, this is becoming more complex, in COPD, asthma and in other diseases too. For instance, great strides have been made in lung cancer and, although it is still known as 'lung cancer' by patients and society, it is clear that 'non-small cell lung cancer' (NSCLC) is a different disease, with different treatments compared with 'small cell lung cancer'. In addition, the identification of an EGFR-mutated NSCLC is a different disease (that requires and responds to a different therapeutic strategy) compared with KRAS-mutated NSCLC. ${ }^{48}$

\section{IN SUMMARY}

The current classification of airways disease may be leading to suboptimal management of a large number of patients and limiting the possibilities of developing novel, personalised therapeutic options. Over the past few years, great strides have been made trying to unravel this complexity through the redefinition of clinical phenotypes, the use of hypothesis-generating techniques and a better understanding of the molecular complexity of airway diseases and their interactions. Moreover, a number of key ongoing studies will, no doubt, move the field forward. Ideally, we would then be able to propose a new taxonomy of airway diseases, leave nineteenth century medicine behind and fully embrace twenty-first century medicine that should be predictive, preventive, personalised and participatory (P4).

I cannot say whether things will get better if we change; what I can say is they must change if they are to get better. (G C Lichtenberg)

\section{Author affiliations}

${ }^{1}$ Program Development, CIRO+, A Centre of Expertise in Chronic Organ Failure, Horn, The Netherlands

${ }^{2}$ Department of Respiratory Medicine, Maastricht University Medical Centre, Maastricht, The Netherlands

${ }^{3}$ Department of General Practice, University Medical Center Groningen, University of Groningen, Groningen, The Netherlands 
${ }^{4}$ Groningen Research Institute for Asthma and COPD (GRIAC), University Medical Centre Groningen, Groningen, The Netherlands

${ }^{5} \mathrm{NIHR}$ Biomedical Research Unit, Centre for Advanced Cardiovascular Imaging, William Harvey Research Institute, Barts Health NHS Trust, The London Chest Hospital, London, UK

${ }^{6}$ Department of Respiratory Medicine, Barts Health NHS Trust, The London Chest Hospital, London, UK

${ }^{7}$ Department of Respiratory and Critical Care Medicine and Ludwig Boltzmann Institute for COPD and Respiratory Epidemiology, Otto Wagner Hospital, Vienna, Austria

${ }^{8}$ Department of Respiratory Medicine, University Hospital of Giessen and Marburg, Marburg, Germany

${ }^{9}$ Department of Medical and Surgical Sciences, Institute of Respiratory Disease, University of Foggia, Foggia, Italy

${ }^{10}$ Pulmonary Department, III medical Centre, Mainz University Hospital, Mainz, Germany

${ }^{11}$ Department of Respiratory Diseases, University of Modena and Reggio Emilia Modena, Modena, Italy

${ }^{12}$ Department of Respiratory Medicine, Allergy and Thoracic Surgery, Institute for Lung Health, Glenfield Hospital, University Hospitals of Leicester NHS Trust, Leicester, UK

${ }^{13}$ Thorax Institute, Hospital Clinic, IDIBAPS, University of Barcelona and FISIB, CIBER Enfermedades Respiratorias (CIBERES), Mallorca, Spain

Acknowledgements We would like to acknowledge Linda Stephens for the organisation of the meetings that provided the basis of this paper.

Contributors LEGWV, JWHK, ISS, RBK, TG, DL, RB, LMF, IDP, NB, EFMW and AA contributed to the discussion and conception of the manuscript. LEGW, JWHK, ISS, RBK, TG, DL and AA wrote the first draft of the manuscript. RB, LMF, IDP, NB, EFMW reviewed the manuscript. LEGW and AA take responsibility for the integrity of the manuscript.

Funding This manuscript is the result of discussions within the European Respiratory Network of Excellence (ERNE), an academic initiative funded by GlaxoSmithKline.

\section{Competing interests None.}

Provenance and peer review Not commissioned; externally peer reviewed.

\section{REFERENCES}

1 Loscalzo J, Barabasi AL. Systems biology and the future of medicine. Wiley Interdiscip Rev Syst Biol Med 2011;3:619-27.

2 Venter JC, Adams MD, Myers EW, et al. The sequence of the human genome. Science 2001;291:1304-51.

3 Loscalzo J, Kohane I, Barabasi AL. Human disease classification in the postgenomic era: a complex systems approach to human pathobiology. Mol Syst Biol 2007:3:124.

4 Vestbo J, Hurd SS, Agusti AG, et al. Global strategy for the diagnosis, management and prevention of chronic obstructive pulmonary disease, GOLD executive summary. Am J Respir Crit Care Med 2013;187:347-65.

5 Global strategy for asthma management and prevention. Updated 2011. http:// www.ginasthma.org/. (accessed 17 Jun 2013).

6 Albert P, Agusti A, Edwards L, et al. Bronchodilator responsiveness as a phenotypic characteristic of established chronic obstructive pulmonary disease. Thorax 2012;67:701-8.

7 O'Byrne PM, Pedersen S, Lamm CJ, et al. Severe exacerbations and decline in lung function in asthma. Am J Respir Crit Care Med 2009;179:19-24.

8 James AL, Palmer $\mathrm{L}$, Kicic $\mathrm{E}$, et al. Decline in lung function in the Busselton Health Study: the effects of asthma and cigarette smoking. Am J Respir Crit Care Med 2005:171:109-14.

9 Eisner MD, Anthonisen N, Coultas D, et al. An official American Thoracic Society public policy statement: Novel risk factors and the global burden of chronic obstructive pulmonary disease. Am J Respir Crit Care Med 2010;182:693-718.

10 Ballarin A, Bazzan E, Zenteno RH, et al. Mast cell infiltration discriminates between histopathological phenotypes of chronic obstructive pulmonary disease. Am J Respir Crit Care Med 2012;186:233-9.

11 Flood-Page PT, Menzies-Gow AN, Kay AB, et al. Eosinophil's role remains uncertain as anti-interleukin-5 only partially depletes numbers in asthmatic airway. Am J Respir Crit Care Med 2003:167:199-204.

12 Pavord ID, Korn S, Howarth P, et al. Mepolizumab for severe eosinophilic asthma (DREAM): a multicentre, double-blind, placebo-controlled trial. Lancet 2012;380:651-9.

13 Bafadhel M, McKenna S, Terry S, et al. Acute exacerbations of chronic obstructive pulmonary disease: identification of biologic clusters and their biomarkers. Am J Respir Crit Care Med 2011;184:662-71.

14 Brightling CE, McKenna S, Hargadon B, et al. Sputum eosinophilia and the short term response to inhaled mometasone in chronic obstructive pulmonary disease. Thorax 2005:60:193-8.
15 Hargreave FE, Nair P. The definition and diagnosis of asthma. Clin Exp Allergy 2009;39:1652-8.

16 Agusti A, Sobradillo P, Celli B. Addressing the complexity of chronic obstructive pulmonary disease: from phenotypes and biomarkers to scale-free networks, systems biology, and P4 medicine. Am J Respir Crit Care Med 2011;183:1129-37.

17 Freimer N, Sabatti C. The human phenome project. Nat Genet 2003;34:15-21.

18 Han MK, Agusti A, Calverley PM, et al. Chronic obstructive pulmonary disease phenotypes: the future of COPD. Am J Respir Crit Care Med 2010;182:598-604.

19 Wenzel SE. Asthma phenotypes: the evolution from clinical to molecular approaches. Nat Med 2012;18:716-25.

20 Stoller JK, Aboussouan LS. A review of alpha1-antitrypsin deficiency. Am J Respir Crit Care Med 2012:185:246-59.

21 Fishman A, Martinez F, Naunheim K, et al. A randomized trial comparing lung-volume-reduction surgery with medical therapy for severe emphysema. $N$ Engl $J$ Med 2003;348:2059-73.

22 Hurst JR, Vestbo J, Anzueto A, et al. Susceptibility to exacerbation in chronic obstructive pulmonary disease. N Engl J Med 2010;363:1128-38.

23 Albert RK, Connett J, Bailey WC, et al. Azithromycin for prevention of exacerbations of COPD. N Engl J Med 2011;365:689-98.

24 Fabbri LM, Calverley PM, Izquierdo-Alonso JL, et al. Roflumilast in moderate-tosevere chronic obstructive pulmonary disease treated with longacting bronchodilators: two randomised clinical trials. Lancet 2009;374:695-703.

25 Calverley PM, Anderson JA, Celli B, et al. Salmeterol and fluticasone propionate and survival in chronic obstructive pulmonary disease. N Engl J Med 2007;356:775-89.

26 Agusti A, Edwards LD, Rennard SI, et al. Persistent systemic inflammation is associated with poor clinical outcomes in COPD: a novel phenotype. PLOS One 2012:7:e37483

27 Haldar P, Pavord ID, Shaw DE, et al. Cluster analysis and clinical asthma phenotypes. Am J Respir Crit Care Med 2008;178:218-24.

28 Wardlaw AJ, Silverman $\mathrm{M}$, Siva $\mathrm{R}$, et al. Multi-dimensional phenotyping: towards a new taxonomy for airway disease. Clin Exp Allergy 2005;35:1254-62.

29 Moore WC, Meyers DA, Wenzel SE, et al. Identification of asthma phenotypes using cluster analysis in the Severe Asthma Research Program. Am J Respir Crit Care Med 2010:181:315-23

30 Lessard A, Turcotte $\mathrm{H}$, Cormier $\mathrm{Y}$, et al. Obesity and asthma: a specific phenotype? Chest 2008:134:317-23.

31 Brusselle GG, Vanderstichele $C$, Jordens $P$, et al. Azithromycin for prevention of exacerbations in severe asthma (AZISAST): a multicentre randomised double-blind placebo-controlled trial. Thorax 2013:68:322-9.

32 Cowan DC, Cowan JO, Palmay R, et al. Effects of steroid therapy on inflammatory cell subtypes in asthma. Thorax 2010;65:384-90.

33 Pavord ID, Wardlaw AJ. The A to E of airway disease. Clin Exp Allergy 2010;40:62-7.

34 Anderson GP. Endotyping asthma: new insights into key pathogenic mechanisms in a complex, heterogeneous disease. Lancet 2008:372:1107-19.

35 Agusti A, Vestbo J. Current controversies and future perspectives in chronic obstructive pulmonary disease. Am I Respir Crit Care Med 2011;184:507-13.

36 Humbert M, Beasley R, Ayres J, et al. Benefits of omalizumab as add-on therapy in patients with severe persistent asthma who are inadequately controlled despite best available therapy (GINA 2002 step 4 treatment): INNOVATE. Allergy 2005;60:309-16

37 Corren J, Lemanske RF, Hanania NA, et al. Lebrikizumab treatment in adults with asthma. N Engl I Med 2011;365:1088-98.

38 Fens $\mathrm{N}$, Roldaan AC, Van der Schee MP, et al. External validation of exhaled breath profiling using an electronic nose in the discrimination of asthma with fixed airways obstruction and chronic obstructive pulmonary disease. Clin Exp Allergy 2011:41:1371-8

39 Weatherall M, Travers J, Shirtcliffe PM, et al. Distinct clinical phenotypes of airways disease defined by cluster analysis. Eur Respir J 2009;34:812-18.

40 Garcia-Aymerich J, Gomez FP, Benet M, et al. Identification and prospective validation of clinically relevant chronic obstructive pulmonary disease (COPD) subtypes. Thorax 2011;66:430-7.

41 Vanfleteren LE, Spruit MA, Groenen M, et al. Clusters of comorbidities based on validated objective measurements and systemic inflammation in patients with chronic obstructive pulmonary disease. Am I Respir Crit Care Med 2013;187:728-35.

42 Celli BR, Cote CG, Marin JM, et al. The body-mass index, airflow obstruction, dyspnea, and exercise capacity index in chronic obstructive pulmonary disease. N Engl J Med 2004:350:1005-12.

43 Puhan MA, Garcia-Aymerich J, Frey M, et al. Expansion of the prognostic assessment of patients with chronic obstructive pulmonary disease: the updated BODE index and the ADO index. Lancet 2009:374:704-11.

44 Jones RC, Donaldson GC, Chavannes NH, et al. Derivation and validation of a composite index of severity in chronic obstructive pulmonary disease: the DOSE Index. Am J Respir Crit Care Med 2009;180:1189-95.

45 Wildfire JJ, Gergen PJ, Sorkness CA, et al. Development and validation of the Composite Asthma Severity Index-an outcome measure for use in children and adolescents. J Allergy Clin Immunol 2012;129:694-701. 
46 Kupczyk M, Wenzel S. U.S. and European severe asthma cohorts: what can they teach us about severe asthma? J Intern Med 2012;272:121-32.

47 Auffray C, Adcock IM, Chung KF, et al. An integrative systems biology approach to understanding pulmonary diseases. Chest 2010;137:1410-16.
48 Brugger W, Triller N, Blasinska-Morawiec M, et al. Prospective molecular marker analyses of EGFR and KRAS from a randomized, placebo-controlled study of erlotinib maintenance therapy in advanced non-small-cell lung cancer. J Clin Oncol 2011;29:4113-20. 